\title{
Timing of retinal neuronal and axonal loss in MS: a longitudinal OCT study
}

\author{
Lisanne J. Balk ${ }^{1} \cdot$ Andrés Cruz-Herranz $^{2}$ • Philipp Albrecht ${ }^{3} \cdot$ Sam Arnow $^{2}$ • \\ Jeffrey M. Gelfand ${ }^{2} \cdot$ Prejaas Tewarie $^{1} \cdot$ Joep Killestein $^{1} \cdot$ Bernard M. J. Uitdehaag $^{1}$ • \\ Axel Petzold ${ }^{1,4,5} \cdot$ Ari J. Green ${ }^{2}$
}

Received: 16 September 2015/Revised: 25 February 2016/Accepted: 11 April 2016/Published online: 3 May 2016

(c) The Author(s) 2016. This article is published with open access at Springerlink.com

\begin{abstract}
The objective of the study was to investigate the timing of central nervous system tissue atrophy in MS by evaluating longitudinal retinal volume changes in a broadly representative cohort with disease duration across the entire arc of disease. In this longitudinal study, 135 patients with MS and 16 healthy reference subjects underwent spectral-domain optical coherence tomography (OCT) at baseline and 2 years later. Following OCT quality control, automated segmentation of the peripapillary retinal nerve fiber layer ( $\mathrm{pRNFL}$ ), macular ganglion cell-inner plexiform layer (mGCIPL) and macular inner nuclear layer (mINL) was performed. Generalized estimation equations were used to analyze longitudinal changes and associations with disease duration and clinical measures. Participants had a median disease duration at baseline of 16.4 years (range $0.1-45.4)$. Nearly half (44\%) of the MS patients had previously experienced MS-related optic neuritis (MSON) more than 6 months prior. The MS patients demonstrated a significant decrease over 2 years of the pRNFL $(-1.1 \mu \mathrm{m}, 95 \%$ CI 1.4-0.7, $p<0.001)$ and mGCIPL $(-1.1 \mu \mathrm{m}, 95 \% \mathrm{CI}-1.4$ to $-0.8, p<0.001)$. This thinning was most pronounced early in the course of
\end{abstract}

Lisanne J. Balk

1.balk@vumc.nl

1 Department of Neurology, MS Centre, VU University Medical Center, Mailbox 7057, 1007 MB Amsterdam, The Netherlands

2 Department of Neurology, University of California San Francisco, San Francisco, USA

3 Department of Neurology, Heinrich-Heine University, Düsseldorf, Germany

4 Moorfields Eye Hospital, City Road, London, UK

5 UCL Institute of Neurology, Queen Square, London, UK disease. These findings were irrespective of previous episodes of MSON. No consistent pattern of change was observed for the mINL $(-0.03 \mu \mathrm{m}, 95 \% \mathrm{CI}-0.2$ to 0.2 , $p=0.795$ ). This longitudinal study demonstrated that injury of the innermost retinal layers is found in MS and that this damage occurs most rapidly during the early stages of disease. The attenuation of atrophy with longer disease duration is suggestive of a plateau effect. These findings emphasize the importance of early intervention to prevent such injury.

Keywords Multiple sclerosis - Optical coherence tomography $\cdot$ Neurodegeneration $\cdot$ Retina $\cdot$ RNFL

\section{Introduction}

Neuroaxonal loss is thought to be the primary substrate underlying irreversible disability in multiple sclerosis (MS) $[1,2]$. However, the timing of neuroaxonal loss in MS is unknown, with injury detected early [3, 4], but more severe atrophy seen at later time stages [5-7]. With renewed interest in developing treatments for progressive MS, and interest in using atrophy measures as a potential biomarker for clinical trials in progressive disease, it is crucially important that the timing of tissue loss in MS is established.

The anterior visual pathway provides a discrete anatomically segregated system to investigate the dynamics of neuroaxonal degeneration in MS [8, 9]. Injury to axons and neurons in the retina can be quantified by retinal spectral-domain optical coherence tomography (SD-OCT). In addition, given the very high degree of reproducibility of OCT, it is a potential method for tracking neuroaxonal loss in MS at the level of the individual patient. Cross-sectional 
studies using SD-OCT have demonstrated evidence of injury to the inner retinal layers in MS, such as the peripapillary retinal nerve fiber layer ( $\mathrm{pRNFL}$ ) and the macular ganglion cell-inner plexiform layer (mGCIPL) [10-14], and established that this injury is correlated with clinical disability [11, 14-16]. Although retinal injury is most severe in the later stages of the disease [17-20], retinal axonal loss has been identified at early stages in the disease course [21-24]. In a large-scale histology study, it was shown that retinal injury not only includes axonal loss (thinning of the pRNFL), but also a reduction in ganglion cell density, demonstrating that inner retinal thinning in MS reflects neuronal and axonal loss [20]. However, the timing and pace of this injury, as with the rest of the central nervous system, remains unknown. Therefore, the main objective of this study was to investigate longitudinal changes in pRNFL, mGCIPL and macular inner nuclear layer (mINL) thickness over a 2 -year period in a broadly representative cohort of patients with a wide range of disease duration in comparison with a healthy reference group. This study was designed to evaluate whether the rate of retinal layer thinning was dependent on disease duration and if the pace of decline was correlated with clinical disability.

\section{Methods}

This study was approved by the Medical Ethical Committees on Human Research of the VU University Medical Center in Amsterdam, the Netherlands, and the University of California, San Francisco Committee on Human Research and is in accordance with the 1964 Declaration of Helsinki. Written informed consent was obtained from all subjects before study inclusion.

\section{Study design and patient population}

In order to investigate the dynamics of retinal layer injury, a broadly representative cohort of patients with MS was included in this longitudinal multi-center observational study. Patients were enrolled from the VU University Medical Center Amsterdam (VUMC) and University of California, San Francisco (UCSF). Additionally, a sample of healthy reference subjects was enrolled at the VU University Medical Center Amsterdam for comparison.

All subjects were required to be between 18 and 80 years of age at the time of their baseline assessment. MS patients were included if they had 'high-risk CIS' (defined as a history of one clinical demyelinating relapse and at least one $\mathrm{T} 2$ hyperintensity typical of demyelination on conventional brain MRI), relapsing remitting MS (RRMS), secondary progressive MS (SPMS) or primary progressive MS (PPMS) at the time of their baseline assessment (as defined by Lublin-Reingold criteria) [25].

All subjects underwent clinical and OCT assessments at baseline and were retested after 24 months (with a 4-month visit window). Disease duration was defined as the time from the first MS symptom. The Expanded Disability Status Scale (EDSS) [26] was obtained by a certified examiner. As potential swelling of the pRNFL during the acute stages of MS related optic neuritis (MSON) may confound OCT measurements, patients were excluded if they had experienced symptomatic MSON 6 months prior to either OCT measurement (baseline or follow-up), according to a standard care protocol [27].

\section{SD-OCT}

Spectral Domain OCT (SD-OCT, Spectralis, Heidelberg Engineering, Heidelberg, Germany) was performed in all subjects at baseline and follow-up. In all scans, eye tracking function was enabled for optimal image registration for averaging and follow-up [28]. For all follow-up scans, automatic scan placement was used with the baseline scan as reference. Data on the pRNFL were obtained using a $12^{\circ}$ ring scan placed around the optic nerve head (Fig. 1A). Data on the macular area (mGCIPL and mINL) were acquired using a macular volume scan $\left(20^{\circ} \times 20^{\circ}\right.$ field, 49 B-scans) centered on the fovea (Fig. 1b).

Scans were excluded from the analyses if they did not fulfill the revised quality control criteria (OSCAR-IB) [29]. In order to assess retinal pathology potentially resulting in exclusion of OCT scans, the medical history of visual symptoms was obtained from all patients and controls. In particular, the assessment of history of symptomatic ON was based on a standard protocol [27] and presence of microcystic macular edema (MME) was assessed according to diagnostic criteria described by Burggraaff et al. [30]. Following this OCT quality control, automated segmentation of the pRNFL, mGCIPL and mINL was performed (Heidelberg Engineering, software version 1.7.1.0). In addition, patients underwent clinical and ophthalmological evaluations for assessment of physical and visual function. Baseline Multiple Sclerosis severity scale (MSSS) scores were calculated for all patients, based on baseline EDSS scores and disease duration.

\section{Statistical analyses}

Differences in disease duration and MSSS scores between the two participating centers were tested using the nonparametric Mann-Whitney $U$ test and the independent sample $T$ test, respectively. Longitudinal changes in retinal layer thickness were treated as absolute change scores $(\Delta$, delta) and relative change scores (percentage change) over 

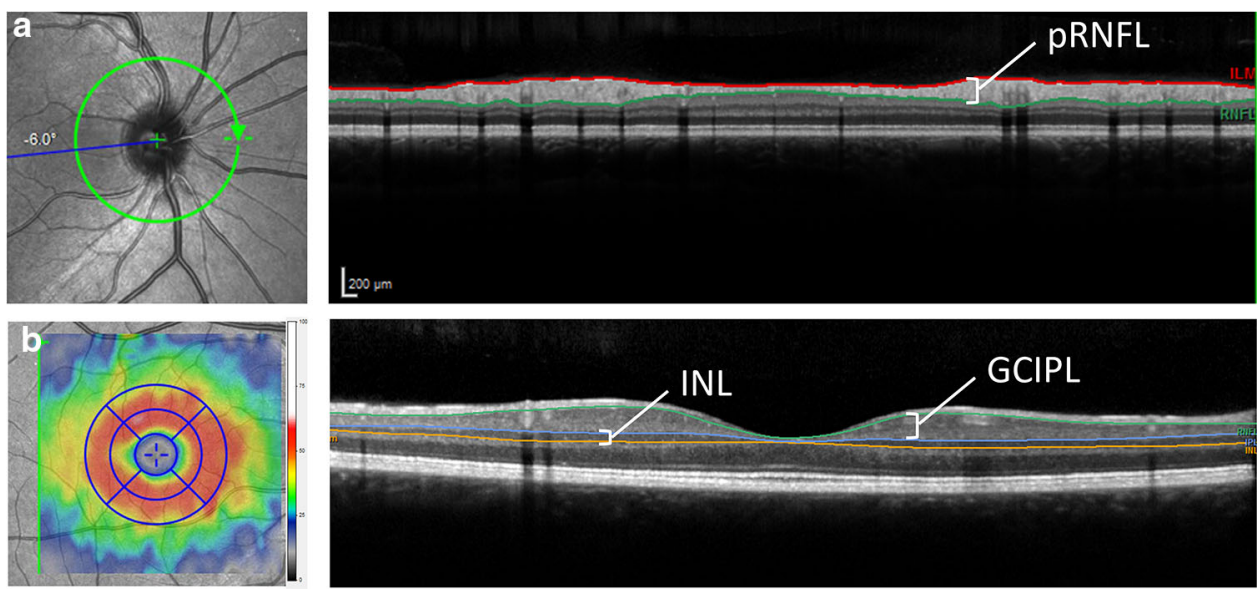

Fig. 1 Scan and segmentation protocol for the OCT scans used in this study. a The peripapillary ring scan $\left(12^{\circ}\right.$ diameter $)$ was used to quantify the global mean retinal nerve fiber layer (pRNFL) thickness. b The macular volume scan $\left(20^{\circ} \times 20^{\circ}\right.$ field, 25 B-scans $)$ was centered on the fovea and used to assess thickness of the macular ganglion cell + inner plexiform layer (mGCIPL) and the inner

the 2-year observation period. Change scores were used to correct for biases caused by potential center-effects. All analyses performed on eye-level (two separate eyes per subject) were done using generalized estimation equations (GEE), with an exchangeable correlation matrix and adjustments for intra-subject inter-eye correlations. Additional adjustments for confounding factors such as age, sex and disease type were performed as indicated. In order to investigate the association with disease duration (defined by time since first symptoms), this variable was categorized into six groups based on the following cut-off values: $<5$ years $\quad(N=31), \quad 5.0-9.9$ years $\quad(N=15)$, 10.0-14.9 years $(N=15), \quad 15.0-19.9$ years $\quad(N=30)$, 20.0-24.9 $(N=17)$ and $\geq 25$ years $(N=26)$.

\section{Results}

A total of 135 MS patients (7 high-risk CIS, 89 RRMS, 26 SPMS, 13 PPMS) were included in this longitudinal study ( $N=65$ from VUMC and $N=70$ from UCSF). At baseline, patients had a median disease duration of 16.4 years, with a range extending from 0.1 to 45.4 years. Although disease duration at baseline was shorter for patients enrolled at UCSF compared with VUMC $(p<0.01$, Fig. 2a), disease severity (reflected by baseline MSSS scores) was similar for patients from UCSF and VUMC ( $p=0.862$, Fig. $2 b)$.

Disease-modifying treatment was used by 58 patients $(43.0 \%)$. Nearly half of all patients had previously experienced a clinically identified episode of unilateral $(30.4 \%)$ or bilateral (most sequential) $(13.3 \%)$ MSON. Presence of MME was not identified in any of the patients, nuclear (mINL). The colored map represents a change in thickness map with a 1, 2.22 and 3.45-mm EDTRS grid of which the eight perimacular sectors were used in this study. The inner $1 \mathrm{~mm}$ circle was excluded from the analysis because of absence of mGCIPL and mINL in the fovea

according to validated criteria [30]. Eight eyes were excluded because of an episode of ON in the 6 months prior to the OCT measurement. None of the included patients had experienced an episode of ON between baseline and follow-up measurement (more than 6 months prior to the follow-up visit). An overview of the clinical and demographic baseline data is shown in Table 1. Additionally, a sample $(N=16)$ of healthy reference subjects was analyzed. Of these 16 subjects, $7(44 \%)$ were female and they had a mean age of 50.7 years (SD 7.1). For all subjects, the mean follow-up period was 24.4 months (SD $1.8)$.

\section{Longitudinal changes in retinal layer thickness}

Table 2 shows the mean individualized changes in pRNFL, mGCIPL and mINL thicknesses over the 2-year observation period. Analyses with all MS eyes included, with a correction for intra-subject inter-eye correlations, demonstrated a significant decrease for both the pRNFL $(-1.1 \mu \mathrm{m})$ and mGCIPL $(-1.1 \mu \mathrm{m}, p<0.001$ for both comparisons) over a 2 -year period. No significant changes were observed for the mINL $(-0.03 \mu \mathrm{m}, p=0.795)$. Importantly, the observed thinning in pRNFL and mGCIPL thickness in patients with MS was significantly larger compared with the healthy reference group $(p=0.015$ and 0.018 , respectively), which showed $-0.5 \mu \mathrm{m}$ thinning for the mGCIPL and $-0.1 \mu \mathrm{m}$ for pRNFL.

Subsequently, analyses stratified for MS eyes with (MSON) and without (MSNON) symptomatic ON showed that longitudinal changes in pRNFL, mGCIPL and mINL thickness were similar for MSON and MSNON eyes. The data demonstrate that previous episodes of MSON did not 


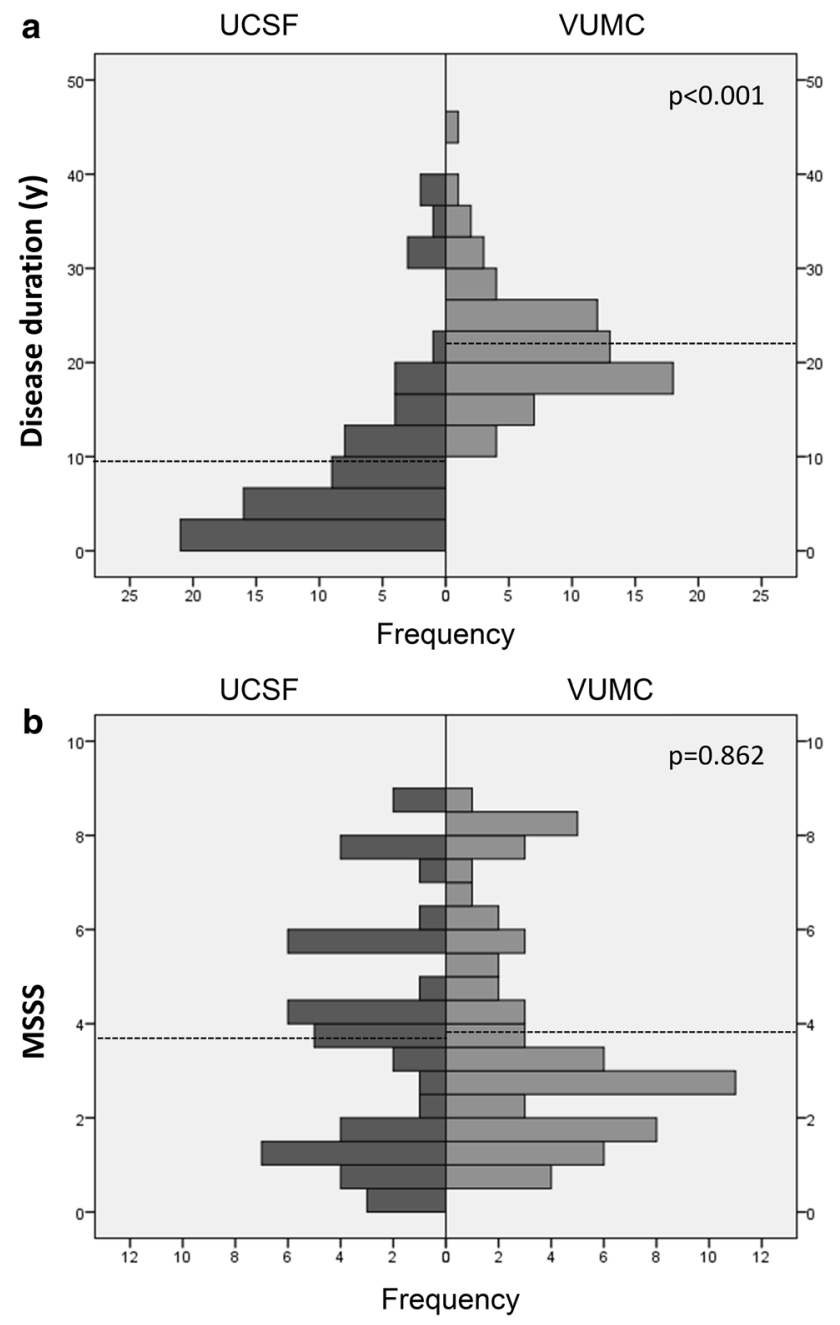

Fig. 2 Center specific distribution of a disease duration (years) and b disease severity (MSSS score). Patients recruited from the VUMC Amsterdam had a significantly longer disease duration [22.0 years (SD 6.5)] compared with patients from UCSF [9.2 years (SD 9.6), $p<0.001]$. Disease severity (MSSS score) was comparable for both centers [UCSF 3.7 (SD 2.5) and VUMC 3.8 (SD 2.4), $p=0.862$ ]. The horizontal dotted lines indicate the mean value per center

significantly affect the rate of thinning during the 2-year observation period for pRNFL $(-1.0 \mu \mathrm{m}$ MSON vs $-1.4 \mu \mathrm{m}$ MSNON, $p=0.125) \mathrm{mGCIPL}(-0.9 \mu \mathrm{m}$ MSON vs $-1.2 \mu \mathrm{m}$ MSNON, $p=0.454)$ or mINL $(-0.04 \mu \mathrm{m}$ MSON vs $-0.06 \mu \mathrm{m}$ MSNON, $p=0.895)$.

When the change scores for the 2-year observation period were stratified according to disease type, a significant difference emerged between relapsing MS and progressive MS (SPMS and PPMS combined) for the pRNFL $(p=0.002)$, but not for the mGCIPL $(p=0.191)$ and mINL $(p=0.540)$. In relapsing MS, significant thinning was observed for the pRNFL $(-1.4 \mu \mathrm{m}, 95 \% \mathrm{CI}-1.8$ to $-1.0)$, which was 14 times more than observed in the reference group $(-0.1 \mu \mathrm{m})$. In SPMS the rate of atrophy
Table 1 Baseline characteristics

\begin{tabular}{|c|c|}
\hline & $\begin{array}{l}\text { MS patients } \\
N=135\end{array}$ \\
\hline $\operatorname{Sex}(N[\%]$ female $)$ & $101(75.0 \%)$ \\
\hline Age (years, mean $[\mathrm{SD}]$ ) & $49.2(\mathrm{SD} 12.1)$ \\
\hline $\begin{array}{l}\text { Disease duration (years, median } \\
\text { [range], IQR) }\end{array}$ & $\begin{array}{l}16.4[0.1-45.9] \\
\text { IQR } 16.3\end{array}$ \\
\hline \multicolumn{2}{|l|}{ Disease type $(N[\%])$} \\
\hline High-risk CIS & $7(5.2 \%)$ \\
\hline $\mathrm{RR}$ & $89(65.9 \%)$ \\
\hline SP & $26(19.3 \%)$ \\
\hline $\mathrm{PP}$ & $13(9.6 \%)$ \\
\hline EDSS score (median [range], IQR) & $\begin{array}{l}3.0[0.0-8.0] \\
\text { IQR } 2.5\end{array}$ \\
\hline MSSS score (mean $[\mathrm{SD}]$ ) & $3.75(\mathrm{SD} 2.4)$ \\
\hline \multicolumn{2}{|l|}{ Disease-modifying treatment } \\
\hline Current & $58(43.0 \%)$ \\
\hline Interferon/glatiramer acetate & $42(72.4 \%)$ \\
\hline Natalizumab & $9(15.5 \%)$ \\
\hline Other & $7(12.1 \%)$ \\
\hline Past & $25(18.5 \%)$ \\
\hline Never & $40(29.6 \%)$ \\
\hline Unknown & $12(8.9 \%)$ \\
\hline \multicolumn{2}{|l|}{ History of optic neuritis $(N[\%])$} \\
\hline None & $72(53.3 \%)$ \\
\hline Unilateral & $41(30.4 \%)$ \\
\hline Bilateral & $18(13.3 \%)$ \\
\hline Unknown & $4(3.0 \%)$ \\
\hline $\begin{array}{l}\text { Time between baseline and follow-up } \\
\text { (months, mean [SD]) }\end{array}$ & $24.4(\mathrm{SD} 1.8)$ \\
\hline \multicolumn{2}{|l|}{ OCT measurements } \\
\hline pRNFL $(\mu \mathrm{m})$ & 83.8 (SD 13.6) \\
\hline mGCIPL $(\mu \mathrm{m})$ & $77.5(\mathrm{SD} 13.8)$ \\
\hline $\operatorname{mINL}(\mu \mathrm{m})$ & 38.9 (SD 3.6) \\
\hline
\end{tabular}

The number (percentage, \%), median [range] and mean [standard deviation, SD] are shown

$C I S$ clinically isolated syndrome, $R R$ relapsing remitting, $S P$ secondary progressive, $P P$ primary progressive, $I Q R$ interquartile range, EDSS Expanded Disability Status Scale, MSSS multiple sclerosis severity score, $p R N F L$ peripapillary retinal nerve fiber layer, $m G C I P L$ macular ganglion cell + inner plexiform layer, $m I N L$ macular inner nuclear layer

over time for the pRNFL was significantly less $(-0.3 \mu \mathrm{m}$, $95 \% \mathrm{CI}-1.0$ to 0.4 ), but still three times larger than in healthy reference subjects. For the mGCIPL, the atrophy rate was slightly higher for the RRMS group $(-1.1 \mu \mathrm{m}$, $95 \%$ CI -1.5 to -0.7$)$ compared to SPMS group [ $-0.8 \mu \mathrm{m}$ (-1.7 to 0.01$), p=0.053]$. Regarding the mINL, neither relapsing nor progressive MS patients showed significant consistent changes over the 2-year observation period across the cohort. 
Table 2 Individualized retinal layer changes over time, stratified by MS patients and healthy controls, MS eyes with and without previous ON and relapsing MS and progressive MS

\begin{tabular}{|c|c|c|c|}
\hline & Absolute change (with $95 \% \mathrm{CI}$ ) & Relative change (with $95 \% \mathrm{CI}$ ) & $p$ value $^{\mathrm{b}}$ \\
\hline \multicolumn{4}{|l|}{ All MS eyes ${ }^{\mathrm{a}}$} \\
\hline pRNFL $\mu \mathrm{m}(N=239$ eyes $)$ & $-1.1(-1.4$ to -0.7$)$ & $-1.3 \%(-1.7$ to -0.9$)$ & $<0.001$ \\
\hline mGCIPL $\mu \mathrm{m}(N=248$ eyes $)$ & $-1.1(-1.4$ to -0.8$)$ & $-1.3 \%(-1.7$ to -0.9$)$ & $<0.001$ \\
\hline $\mathrm{mINL} \mu \mathrm{m}(N=248$ eyes $)$ & $-0.03(-0.2$ to 0.2$)$ & $-0.03 \%(-0.6$ to 0.5$)$ & 0.795 \\
\hline \multicolumn{4}{|l|}{ Healthy references } \\
\hline pRNFL $(N=31$ eyes $)$ & $-0.1(-0.8$ to 0.6$)$ & $-0.1 \%(-0.8$ to 0.5$)$ & 0.797 \\
\hline mGCIPL ( $N=30$ eyes $)$ & $-0.5(-0.9$ to -0.1$)$ & $-0.5 \%(-1.0$ to 0.0$)$ & 0.021 \\
\hline $\operatorname{mINL}(N=30$ eyes $)$ & $+0.4(-0.1$ to 0.9$)$ & $+1.0 \%(0.0$ to 2.2$)$ & 0.082 \\
\hline \multicolumn{4}{|l|}{ MSNON eyes } \\
\hline pRNFL $\mu \mathrm{m}(N=168$ eyes $)$ & $-1.0(-1.4$ to -0.5$)$ & $-1.1 \%(-1.5$ to -0.6$)$ & $<0.001$ \\
\hline mGCIPL $\mu \mathrm{m}(N=174$ eyes $)$ & $-0.9(-1.2$ to -0.6$)$ & $-1.1 \%(-1.5$ to -0.7$)$ & $<0.001$ \\
\hline $\mathrm{mINL} \mu \mathrm{m}(N=174$ eyes $)$ & $-0.04(-0.3$ to 0.2$)$ & $-0.03 \%(-0.8$ to 0.7$)$ & 0.769 \\
\hline \multicolumn{4}{|l|}{ MSON eyes } \\
\hline pRNFL $\mu \mathrm{m}(N=64$ eyes $)$ & $-1.4(-2.0$ to -0.7$)$ & $-1.7 \%(-2.5$ to -1.0$)$ & $<0.001$ \\
\hline mGCIPL $\mu \mathrm{m}(N=74$ eyes $)$ & $-1.2(-2.0$ to -0.5$)$ & $-1.7 \%(-2.8$ to -0.7$)$ & $<0.001$ \\
\hline $\operatorname{mINL} \mu \mathrm{m}(N=74$ eyes $)$ & $-0.06(-0.5$ to 0.3$)$ & $-0.1 \%(-1.1$ to 0.9$)$ & 0.862 \\
\hline \multicolumn{4}{|l|}{ RRMS eyes } \\
\hline pRNFL $(N=170)$ & $-1.4(-1.8$ to -1.0$)$ & $-1.6 \%(-2.1$ to -1.1$)$ & $<0.001$ \\
\hline mGCIPL $(N=176)$ & $-1.1(-1.5$ to -0.7$)$ & $-1.4 \%(-1.9$ to -0.9$)$ & $<0.001$ \\
\hline $\operatorname{mINL}(N=176)$ & $-0.08(-0.3$ to 0.2$)$ & $-0.15 \%(-0.8$ to 0.5$)$ & 0.486 \\
\hline \multicolumn{4}{|l|}{ SPMS eyes } \\
\hline pRNFL $(N=48)$ & $-0.3(-1.0$ to 0.4$)$ & $-0.3(-1.2$ to 0.6$)$ & 0.395 \\
\hline mGCIPL $(N=48)$ & $-0.8(-1.7$ to 0.01$)$ & $-1.2(-2.4$ to -0.0$)$ & 0.053 \\
\hline $\operatorname{mINL}(N=48)$ & $-0.1(-0.5$ to 0.3$)$ & $-0.3(-1.3$ to 0.8$)$ & 0.598 \\
\hline \multicolumn{4}{|l|}{ PPMS eyes } \\
\hline pRNFL $(N=21)$ & $+0.01(-1.0$ to 1.0$)$ & $+0.1 \%(-1.1$ to 1.2$)$ & 0.987 \\
\hline mGCIPL $(N=24)$ & $-0.4(-1.5$ to 0.6$)$ & $-0.5(-1.7$ to 0.6$)$ & 0.448 \\
\hline $\operatorname{mINL}(N=24)$ & $0.8(-0.3$ to 1.8$)$ & $+2.0(-0.7$ to 4.7$)$ & 0.155 \\
\hline
\end{tabular}

Bold values indicate $p<0.05$

$p R N F L$ peripapillary retinal nerve fiber layer, $m G C I P L$ macular ganglion cell + inner plexiform layer, $m I N L$ macular inner nuclear layer, $O N$ optic neuritis, RRMS relapsing remitting MS, SPMS secondary progressive MS, PPMS primary progressive MS

${ }^{a}$ Scans which did not fulfill quality control criteria (OSCAR-IB) were excluded from the analyses

${ }^{\mathrm{b}}$ Unadjusted GEE analysis (only intercept models) using the absolute change scores

\section{Changes in retinal layer thickness are more pronounced early in the course of disease}

Thinning of the pRNFL and mGCIPL was significantly related to disease duration $[\beta 0.05,95 \%$ CI $0.01-0.09$, $p=0.012$ and $\beta 0.06,95 \%$ CI $0.03-0.10, p=0.001$, respectively (GEE, adjusted for inter-eye correlation, optic neuritis and sex)]. Because change scores were negative (which indicates thinning), the positive regression coefficients demonstrate that with a longer disease duration, the rate of thinning becomes smaller. More specifically, for every additional year of disease duration, the rate of thinning over a 2-year period, decreases by $0.05 \mu \mathrm{m}$ for the pRNFL and $0.06 \mu \mathrm{m}$ for the mGCIPL. Changes in mINL thickness did not show a relationship with disease duration. The associations between retinal layer thinning and disease duration are shown in Fig. 3.

To investigate the association with disease duration in more detail, disease duration was categorized into six groups. Figure 4 shows the changes in retinal layer thickness for all disease duration categories. Thinning of the pRNFL and mGCIPL over the 2-year observation period was most pronounced in patients with a shorter disease duration. For the mINL, no such pattern was observed.

\section{Discussion}

This study showed that over a 2-year observation period, significant thinning of the pRNFL and mGCIPL was observed throughout the course of the disease, but that 
a

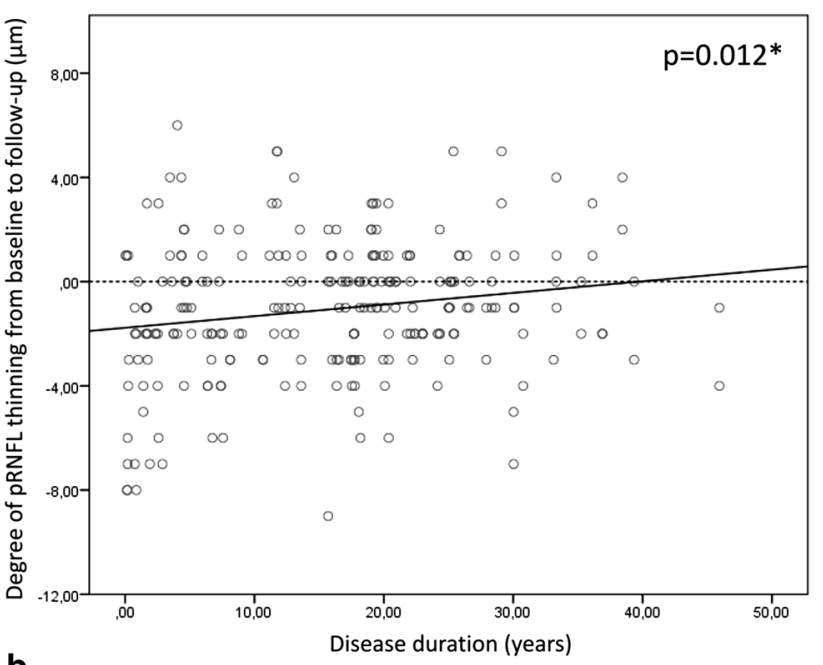

b

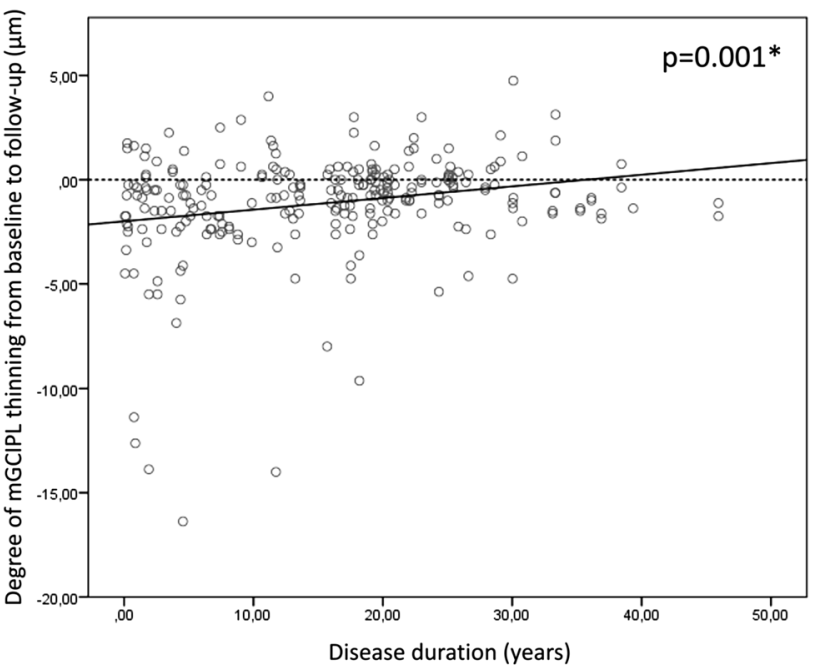

C

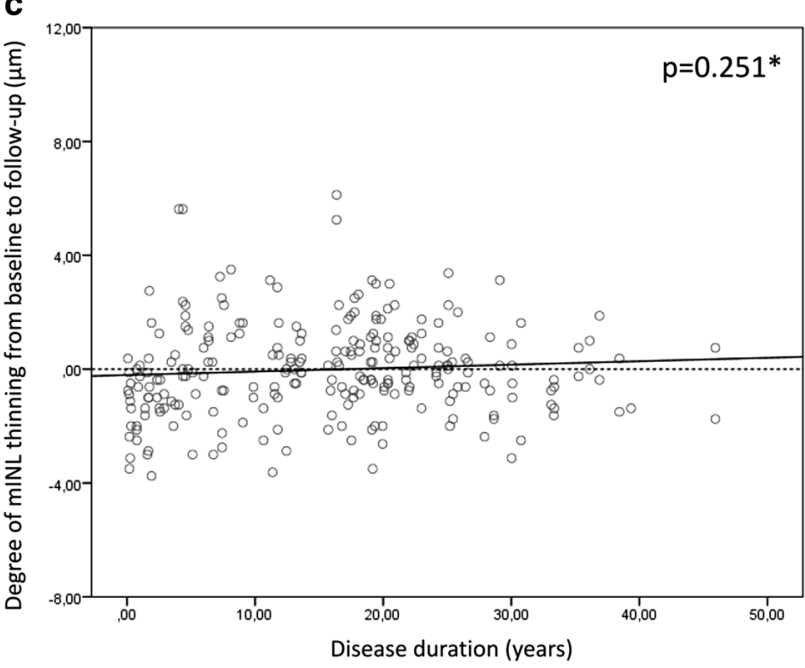

these changes were significantly more pronounced early in the disease course. The attenuation of atrophy with longer disease duration is suggestive of a plateau effect. Our
4Fig. 3 Association between disease duration and change in retinal layer thickness. Thinning of the pRNFL $\mathbf{a}$ and mGCIPL $\mathbf{b}$ is significantly associated with disease duration. For the mINL (C), no significant association was observed. * GEE, adjusted for inter-eye correlation, optic neuritis, sex

findings argue in favor of significant progressive neuronal and axonal injury occurring during the earliest stages of MS, providing support for early intervention to prevent or forestall this injury.

Establishing the timing of neuroaxonal loss in MS is crucial as we explore methods for assessing potential therapeutics meant to treat progressive disease and prevent this decline. It has been presumed in much of the literature that neuroaxonal loss is the substrate underlying volume loss on brain MRI. However, routinely used sequences on brain MRI lack specificity in this regard, making the retina an attractive additional site to monitor. Use of atrophy as a biomarker in progressive MS trials is dependent on the assumption that continued atrophy accumulates in the progressive phase of disease. This study helps to establish that the rate of atrophy in progressive disease may be lower and helps inform timing and sample size calculations for trials using OCT as an outcome.

In the present study, a longitudinal investigation of three retinal layers was performed, the pRNFL, mGCIPL and mINL. Firstly, our data showed significant thinning of the pRNFL $(-1.1 \mu \mathrm{m})$, over 10 times more than observed in healthy reference subjects $(-0.1 \mu \mathrm{m})$. This is in line with previously reported findings of longitudinal changes in pRNFL thickness obtained using time domain OCT [16, $18,31]$. In more recent studies using spectral-domain OCT, Narayanan and colleagues reported an annualized decrease in pRNFL of $-1.49 \mu \mathrm{m}$ for MSNON eyes, and $-1.27 \mu \mathrm{m}$ for MSON eyes, but they did not assess for the impact of disease duration or assess a cohort with a broad representation of disease durations [32]. Saidha and colleagues, however, reported a considerably smaller yearly decrease of $-0.36 \mu \mathrm{m}$ (MSON and MNON eyes together) [33].

Second, regarding the mGCIPL, we reported significant thinning over a 2-year observation period $(-1.1 \mu \mathrm{m})$. This decrease in mGCIPL thickness, more than twice as much as observed in the reference population, was similar for MSON and MSNON eyes. In a recent longitudinal studies, Narayanan and colleagues reported comparable findings, with a yearly mGCIPL decrease of $-0.53 \mu \mathrm{m}$ in MSON and -0.49 in MSNON eyes [32], whereas Saidha et al. reported an annual change of $-0.34 \mu \mathrm{m}$ (MSON and MSNON together) [33].

Third, with respect to the mINL, the present study showed no consistent changes in patients with MS across the cohort, independent of disease duration or disease type. To our knowledge, no other longitudinal studies have 
a
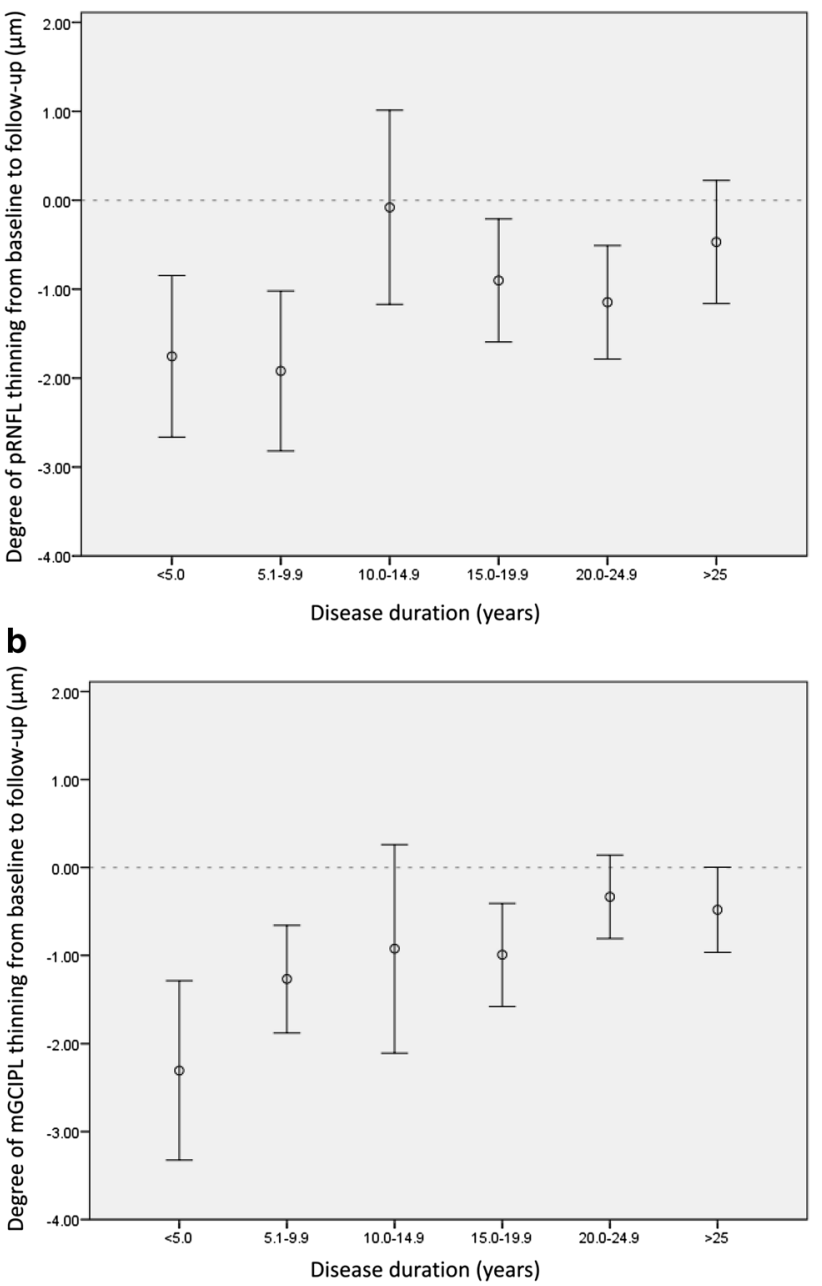

C

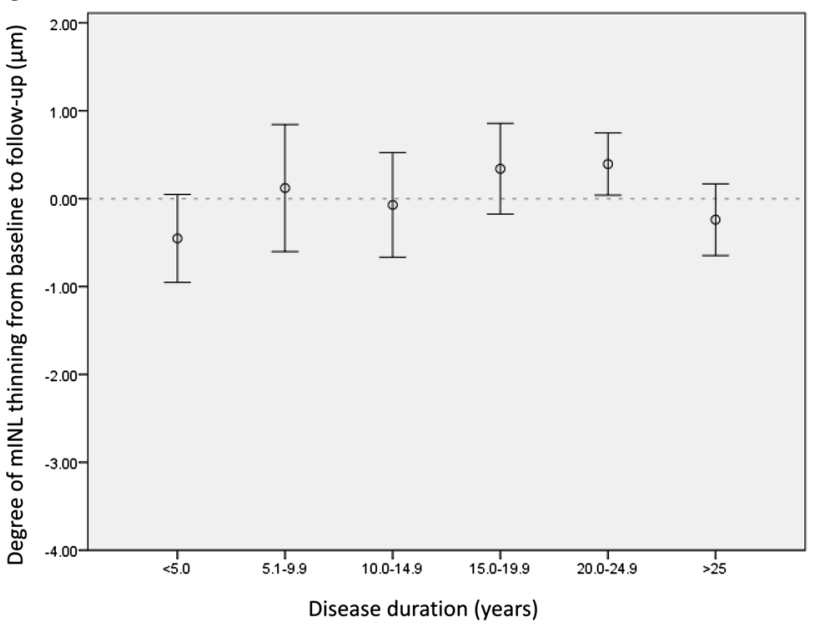

investigated changes in mINL thickness. Interestingly, in human retinal postmortem samples, extensive loss of neurons in the mINL has been demonstrated after about 20 years of disease duration [20]. This discrepancy between in vivo and in vitro data would argue in favor of
Fig. 4 Mean change and $95 \%$ CIs (error bars) in pRNFL (a), mGCIPL (b), and mINL (c) per disease duration group. pRNFL and mGCIPL thickness changes were dependent on disease duration, as changes were most pronounced early in the disease course. The horizontal dotted reference line indicates no change from baseline [group sizes: $<5$ years $(N=31), \quad 5.0-9.9$ years $\quad(N=15)$, 10.0-14.9 years $(N=15), \quad 15.0-19.9$ years $(N=30), \quad 20.0-24.9$ $(N=17)$ and $\geq 25$ years $(N=26)]$

mINL thinning as being a dynamic process, instead of a constant process presenting a steady decrease during the disease course. This hypothesis is further strengthened by the reported increase in mINL thickness associated with MME. The occurrence of MME in MS [34], is characterized by the presence of cystic areas of hyporeflectivity in the mINL of the retina and it was shown that INL thickness changes were dynamic and were associated with increased disability [35]. Since none of the subjects experienced MME during the study, the present data do not allow for interpretation of the direct effect of MME on changes in mINL thickness, but we do not rule out the possibility of dynamic changes at a time scale not measured within the present study.

Taken together, available longitudinal literature consistently demonstrates progressive loss of pRNFL and mGCIPL in patients with MS. It was, however, previously unclear whether this inner retinal layer damage is a continuous process (as is often assumed with linear modeling), or if the underlying process is dependent on the stage of the disease. Previous studies have shown that retinal axonal injury was already present early in the disease course of MS [20-23]. Although the present data confirm this finding, we have also conclusively shown that the rate of pRNFL and mGCIPL thinning is dependent on disease duration and is greatest in early phases of the disease. Importantly, previous data on annual change of inner retinal layer thickness are inconsistent. Given the findings of the present study, we believe that annual change is clearly influenced by disease duration. Therefore, the inconsistent findings reported in literature are probably partly caused by unaccounted differences in disease duration between study cohorts.

Furthermore, whereas pRNFL and mGCIPL thinning were both present in eyes of patients with relapsing MS, the eyes of SPMS patients only showed substantial thinning of the mGCIPL. This finding is in concurrence with a previous longitudinal study by Henderson and colleagues, who reported no significant decrease in pRNFL thickness after an average follow-up of over 18 months in a cohort of progressive MS patients [19]. This finding suggests that although both neuronal and axonal injuries occur in the relapsing phase of the disease, only subtle neuronal damage continues in the progressive phase. Notwithstanding, this finding should be interpreted with caution, as the effect was 
[although statistically significant $(p=0.015)$ ], only modestly stronger than observed in healthy references.

The present data support the large body of evidence on the effects of previous episode of MSON on pRNFL and mGCIPL thickness. Consistent with many other studies $[10,36-38]$, we demonstrated that the eyes of patients who experienced previous episodes of MSON showed significantly more damage of both inner layers at baseline, compared to unaffected (MSNON) eyes. The longitudinal data of the present study, however, extend to these findings, as we demonstrated that although the MSON eyes showed significantly more injury at baseline, the rate of thinning over time was similar for $\mathrm{ON}$ affected and unaffected eyes. Consequently, while prior episodes of MSON act as a major confounder in cross-sectional studies, its effect seems considerably less important in longitudinal investigations.

A major strength of the present study was the longitudinal design. Although cross-sectional studies have provided very useful and important data on retinal layer injury in MS, the longitudinal design of the present study, together with the very broadly representative cohort of patients, allowed for interpretation of the dynamics of retinal changes in different stages of MS. Although the 2-year observation period of the present study showed significant changes, the absolute changes were relatively small. Therefore, our current studies are focused on longer observation periods (3-5 years) with repeated OCT and clinical assessments. Our study may have several limitations. First, the control group was relatively small. Although this group was fairly homogeneous and similar to the patient population, a larger sample would increase statistical power. Similarly, although patients with a disease duration across the entire arc of disease were included, those with a progressive disease course (SPMS and PPMS) may have been underpowered, as the majority of patients had relapsing remitting MS. Second, extensive low-contrast visual acuity and color vision testing was not included in the present study. Both measures would provide important information on visual functioning and should be included in future studies [27].

In summary, this study showed that over a 2-year observation period, significant thinning of the pRNFL and mGCIPL, but not the mINL, was observed in patients with MS throughout the course of disease. These changes in pRNFL and mGCIPL were more pronounced early in the disease course. The attenuation of atrophy with longer disease duration suggests that the earliest phases of disease provide the optimal time to prevent permanent neuroaxonal injury.

Acknowledgments We would like to thank the Dutch MS Research foundation for granting Dr. L.J. Balk with a personal travel grant. We also would like to thank all patients and healthy reference subjects for participating in this study.

\section{Compliance with ethical standards}

Conflicts of interest LB reports grants from the Dutch MS Research Foundation, during the conduct of the study and the VUmc MS Centre reports grants for OCT projects from TEVA. AC, PT and SA report no disclosures. PA reports grants and personal fees from Biogen Idec, grants, personal fees and non-financial support from Novartis, grants from Merz Pharmaceuticals, personal fees and nonfinancial support from Teva, non-financial support from Ipsen, outside the submitted work. JG reports grants from NIH KL2TR000143, personal fees from MedImmune, support from Quest Diagnostics for work on a Dementia Care Pathway, personal fees from Medical-Legal Consulting, outside the submitted work. JK reports grants and personal fees from Biogen Idec, Novartis, Merck Serono, TEVA, Genzyme, Bayer Schering Pharma Glaxo Smith Kline, outside the submitted work. BU reports personal fees from Novartis, Merck Serono, Biogen Idec and Danone Research, outside the submitted work. AP reports grants from Dutch MS Society, grants from VKC foundation, during the conduct of the study; and is a member of the steering committee of the OCTiMS study by Novartis. AG reports personal fees from Roche, Accorda, Mylan Pharma, grants from Rachleff Family, NMSS, Novartis, UCSF CTSI, That Man May See, Robert Dale Family and Medimmune, outside the submitted work.

Open Access This article is distributed under the terms of the Creative Commons Attribution 4.0 International License (http://crea tivecommons.org/licenses/by/4.0/), which permits unrestricted use, distribution, and reproduction in any medium, provided you give appropriate credit to the original author(s) and the source, provide a link to the Creative Commons license, and indicate if changes were made.

\section{References}

1. Trapp BD, Nave KA (2008) Multiple sclerosis: an immune or neurodegenerative disorder? Annu Rev Neurosci 31:247-269

2. Petzold A (2014) Neurodegeneration and multiple sclerosis. In: Galimberti D, Scaroini E (eds) Neurodegenerative diseases. Springer, London, pp 227-245

3. Dalton CM, Chard DT, Davies GR, Miszkiel KA, Altmann DR, Fernando K et al (2004) Early development of multiple sclerosis is associated with progressive grey matter atrophy in patients presenting with clinically isolated syndromes. Brain 127:1101-1107

4. De SN, Matthews PM, Filippi M, Agosta F, De LM, Bartolozzi ML et al (2003) Evidence of early cortical atrophy in MS: relevance to white matter changes and disability. Neurology 8 60(7):1157-1162

5. Calabrese M, Gallo P (2009) Magnetic resonance evidence of cortical onset of multiple sclerosis. Mult Scler 15(8):933-941

6. Fisher E, Lee JC, Nakamura K, Rudick RA (2008) Gray matter atrophy in multiple sclerosis: a longitudinal study. Ann Neurol 64(3):255-265

7. Fisniku LK, Chard DT, Jackson JS, Anderson VM, Altmann DR, Miszkiel KA et al (2008) Gray matter atrophy is related to longterm disability in multiple sclerosis. Ann Neurol 64(3):247-254

8. Costello F (2013) The afferent visual pathway: designing a structural-functional paradigm of multiple sclerosis. ISRN Neurol 2013:134858

9. Martinez-Lapiscina E, Sanchez-Dalmau B, Fraga-Pumar E, OrtizPerez S, Tercero-Uribe A, Torres-Torres R et al (2014) The visual 
pathway as a model to understand brain damage in multiple sclerosis. Mult Scler 20(13):1678-1685

10. Balk LJ, Twisk JW, Steenwijk MD, Daams M, Tewarie P, Killestein $\mathrm{J}$ et al (2014) A dam for retrograde axonal degeneration in multiple sclerosis? J Neurol Neurosurg Psychiatry 85(7):782-789

11. Albrecht $P$, Ringelstein M, Muller AK, Keser N, Dietlein T, Lappas A et al (2012) Degeneration of retinal layers in multiple sclerosis subtypes quantified by optical coherence tomography. Mult Scler 18(10):1422-1429

12. Gabilondo I, Martinez-Lapiscina EH, Martinez-Heras E, FragaPumar E, Llufriu S, Ortiz S et al (2014) Trans-synaptic axonal degeneration in the visual pathway in multiple sclerosis. Ann Neurol 75(1):98-107

13. Saidha S, Sotirchos ES, Oh J, Syc SB, Seigo MA, Shiee N et al (2013) Relationships between retinal axonal and neuronal measures and global central nervous system pathology in multiple sclerosis. JAMA Neurol 70(1):34-43

14. Tatrai E, Simo M, Iljicsov A, Nemeth J, Debuc DC, Somfai GM (2012) In vivo evaluation of retinal neurodegeneration in patients with multiple sclerosis. PLoS One 7(1):e30922

15. Sakai RE, Feller DJ, Galetta KM, Galetta SL, Balcer LJ (2011) Vision in multiple sclerosis: the story, structure-function correlations, and models for neuroprotection. J Neuroophthalmol 31(4):362-373

16. Sepulcre J, Murie-Fernandez M, Salinas-Alaman A, GarciaLayana A, Bejarano B, Villoslada P (2007) Diagnostic accuracy of retinal abnormalities in predicting disease activity in MS. Neurology 68(18):1488-1494

17. Trip SA, Schlottmann PG, Jones SJ, Altmann DR, Garway-Heath DF, Thompson AJ et al (2005) Retinal nerve fiber layer axonal loss and visual dysfunction in optic neuritis. Ann Neurol 58(3):383-391

18. Talman LS, Bisker ER, Sackel DJ, Long DAJ, Galetta KM, Ratchford JN et al (2010) Longitudinal study of vision and retinal nerve fiber layer thickness in multiple sclerosis. Ann Neurol 67(6):749-760

19. Henderson APD, Trip SA, Schlottmann PG, Altmann DR, Garway-Heath DF, Plant GT et al (2010) A preliminary longitudinal study of the retinal nerve fiber layer in progressive multiple sclerosis. J Neurol 257(7):1083-1091

20. Green AJ, McQuaid S, Hauser SL, Allen IV, Lyness R (2010) Ocular pathology in multiple sclerosis: retinal atrophy and inflammation irrespective of disease duration. Brain 133(Pt 6):1591-1601

21. Gelfand JM, Goodin DS, Boscardin WJ, Nolan R, Cuneo A, Green AJ (2012) Retinal axonal loss begins early in the course of multiple sclerosis and is similar between progressive phenotypes. PLoS One 7(5):e36847

22. Young KL, Brandt AU, Petzold A, Reitz LY, Lintze F, Paul F et al (2013) Loss of retinal nerve fibre layer axons indicates white but not grey matter damage in early multiple sclerosis. Eur $\mathbf{J}$ Neurol 20(5):803-811

23. Oberwahrenbrock T, Ringelstein M, Jentschke S, Deuschle K, Klumbies K, Bellmann-Strobl J et al (2013) Retinal ganglion cell and inner plexiform layer thinning in clinically isolated syndrome. Mult Scler 19(14):1887-1895
24. Huhn K, Lammer R, Oberwahrenbrock T, Lammer A, Waschbisch A, Gosar D et al (2014) Optical coherence tomography in patients with a history of juvenile multiple sclerosis reveals early retinal damage. Eur J Neurol 22(1):86-92

25. Lublin FD, Reingold SC (1996) Defining the clinical course of multiple sclerosis: results of an international survey. National Multiple Sclerosis Society (USA) Advisory Committee on Clinical Trials of New Agents in Multiple Sclerosis. Neurology 46(4):907-911

26. Kurtzke JF (1983) Rating neurologic impairment in multiple sclerosis: an expanded disability status scale (EDSS). Neurology 33(11):1444-1452

27. Petzold A, Wattjes MP, Costello F, Flores-Rivera J, Fraser CL, Fujihara K et al (2014) The investigation of acute optic neuritis: a review and proposed protocol. Nat Rev Neurol 10(8):447-458

28. Balk LJ, Petzold A (2013) Influence of the eye-tracking-based follow-up function in retinal nerve fiber layer thickness using fourier-domain optical coherence tomography. Invest Ophthalmol Vis Sci 54(4):3045

29. Schippling S, Balk L, Costello F, Albrecht P, Balcer L, Calabresi $P$ et al (2014) Quality control for retinal OCT in multiple sclerosis: validation of the OSCAR-IB criteria. Mult Scler 21(2):163-170

30. Burggraaff MC, Trieu J, de Vries-Knoppert WA, Balk L, Petzold A (2014) The clinical spectrum of microcystic macular edema. Invest Ophthalmol Vis Sci 55(2):952-961

31. Costello F, Hodge W, Pan YI, Freedman M, DeMeulemeester C (2009) Differences in retinal nerve fiber layer atrophy between multiple sclerosis subtypes. J Neurol Sci 281(1-2):74-79

32. Narayanan D, Cheng H, Bonem KN, Saenz R, Tang RA, Frishman LJ (2014) Tracking changes over time in retinal nerve fiber layer and ganglion cell-inner plexiform layer thickness in multiple sclerosis. Mult Scler 20(10):1331-1341

33. Saidha S, Al-Louzi O, Ratchford JN, Bhargava P, Oh J, Newsome SD et al (2015) Optical coherence tomography reflects brain atrophy in MS: a four year study. Ann Neurol. doi:10.1002/ana. 24487

34. Gelfand JM, Nolan R, Schwartz DM, Graves J, Green AJ (2012) Microcystic macular oedema in multiple sclerosis is associated with disease severity. Brain 135(Pt 6):1786-1793

35. Saidha S, Sotirchos ES, Ibrahim MA, Crainiceanu CM, Gelfand JM, Sepah YJ et al (2012) Microcystic macular oedema, thickness of the inner nuclear layer of the retina, and disease characteristics in multiple sclerosis: a retrospective study. Lancet Neurol 11(11):963-972

36. Petzold A, de Boer JF, Schippling S, Vermersch P, Kardon R, Green A et al (2010) Optical coherence tomography in multiple sclerosis: a systematic review and meta-analysis. Lancet Neurol 9(9):921-932

37. Syc SB, Saidha S, Newsome SD, Ratchford JN, Levy M, Ford E et al (2012) Optical coherence tomography segmentation reveals ganglion cell layer pathology after optic neuritis. Brain $135(\mathrm{Pt}$ 2):521-533

38. Gabilondo I, Martinez-Lapiscina EH, Fraga-Pumar E, Ortiz-Perez $\mathrm{S}$, Torres-Torres R, Andorra M et al (2015) Dynamics of retinal injury after acute optic neuritis. Ann Neurol 77(3):517-528 